\title{
DAS EUROPÄISCHE RAUMENTWICKLUNGSKONZEPT ALS GRUNDLAGE FÜR GRENZÜBERSCHREITENDE KOOPERATIONEN
}

\author{
Friedrich M. Zimmermann*
}

\author{
EVROPSKI KONCEPT PROSTORSKEGA RAZVOJA KOT TMELJ PREKOMEJNEGA \\ SODELOVANJA \\ Izvleček \\ UDK 339.92(4-04)
}

Prispevek poroča o ciljih in strategijah bruseljskega Evropskega koncepta prostorskega razvoja (EUREK). Avtor osvetli nekatere možnosti, ki jih ta strateški koncept ponuja in priporoča skupno načrtovanje razvoja na obmejnih območjih, tako partnerskih držav Evropske zveze kot njih in kandidatk za priključitev. Prostorski načrti, ki bodo upoštevali danosti in komplementarnost območij ob meji bodo deležni pomoči, ki jo nakazuje obravnavani strateški program $E U$.

Ključne besede: Evropska zveza, EUREK, prostorsko načrtovanje, širjenje EU, obmejna območja, prekomejno sodelovanje.

\section{THE EUROPEAN CONCEPT OF SPATIAL DEVELOPMENT AS THE BASE FOR TRANSBORDER COOPERATIONS}

\begin{abstract}
This is a report on goals and projected strategies of The European Concept of Spatial Development (EUREK) which was launched in Brussels, in 1999. The author points out selected visions of the programme and calls for a concentrated action of the candidates to join the European Union and the member states themselves to develop several transborder cooperations projects. The complementarity in the border regions and the planning of such developments across borders will be supported by the EUREK itself.
\end{abstract}

Key words: European Union, EUREK, spatial planning, the enlargement of the EU, border regions, transborder cooperation.

\footnotetext{
* Dr., Institut für Geographie und Raumforschung, Universität Graz, Heinrichstraße 36, A-8010 Graz, Österreich
} 


\section{VORBEMERKUNGEN}

Der Ausschuss für Raumentwicklung hat beim Treffen der für die Raumordnung zuständigen Minister der Europäischen Union in Potsdam am 10. und 11. Mai 1999 das EUROPÄISCHE RAUMENTWICKLUNGS-KONZEPT (EUREK) - "Auf dem Weg zu einer räumlich ausgewogenen und nachhaltigen Entwicklung der EU" vorgelegt. Dieses Dokument wurde beim oben angeführten Treffen verabschiedet und bildet somit den Europäischen Rahmen für räumliche Entwicklungen. Dieses Dokument besteht aus 2 Teilen, die wesentlichen Kernbereiche lassen sich wie folgt charakterisieren:

1. Teil A:

- Für eine ausgewogene und nachhaltige Entwicklung des Territoriums der EU: Der Beitrag ung.

- Ausgewählte Programme und Leitbilder für eine integrierte Raumentwicklung.

- Basisdaten für die Beitrittsstaaten und Mitgliedsstaaten.

Bei der Definition des räumlichen Ansatzes auf europäischer Ebene wird festgehalten, dass das charakteristische Element Europas seine auf engem Raum konzentrierte kulturelle Vielfalt ist. Diese Vielfalt ist einer der bedeutendsten Entwicklungsfaktoren der Europäischen Union und muss im Zuge der Integration bewahrt und geschützt werden. Daher sind die Politiken, die Einfluss auf die Raumgestaltung haben, darauf auszurichten, dass sie zur Bereicherung der Lebensqualität beitragen und nicht zur Vereinheitlichung. Mit dem Zusammenwachsen Europas verlieren die Grenzen zunehmend ihren trennenden Charakter, es entstehen intensivere Interaktionen und Verflechtungen zwischen Städten und Regionen der Mitgliedstaaten aber auch der Beitrittskandidaten. Regionale, nationale und gemeinschaftliche Vorhaben und Projekte haben oftmals über die Staatsgrenzen hinausgehende Auswirkungen auf die Raumstruktur und Raumentwicklung. Mit der Weiterentwicklung Europas müssen die räumlichen Wirkungen frühzeitig berücksichtigt werden, um Negativeffekte von Grenzen zu reduzieren. Auch wenn Regionen, Städte oder Gemeinden bereits begonnen haben, grenzüberschreitend zu kooperieren, so stehen sie dennoch miteinander in Konkurrenz um Betriebsansiedlungen, Arbeitsplätze, Infrastruktur etc.

Die langfristigen räumlichen Entwicklungen in der Europäischen Union werden vor allem durch drei Faktoren beeinflusst:

- Die fortschreitende wirtschaftliche Integration und die damit verbundene verstärkte Kooperation zwischen den Mitgliedstaaten.

- Die wachsende Bedeutung der lokalen und regionalen Gebietskörperschaften und ihre Rolle bei der Raumentwicklung. 
- $\quad$ Die zu erwartende Erweiterung der Europäischen Union und die Entwicklung der Beziehungen zu ihren Nachbarn.

Daraus ist abzuleiten, dass Entwicklungsvorhaben von Mitgliedsstaaten oder aber Beitrittskandidaten sich am besten daran orientieren, gemeinsam festgelegte Ziele zur räumlichen Entwicklung zu definieren. Die nationalen Politiken und die Fachpolitiken der Europäischen Union bedürfen räumlich definierter Leitbilder. Diese werden mit dem europäischen Raumentwicklungskonzept vorgelegt. Dieses Raumentwicklungskonzept bietet die Möglichkeit, sektororientierte Blickweisen auf die Entwicklung des gesamten europäischen Territoriums hin auszurichten und dabei individuelle Entwicklungspotentiale in Regionen zu berücksichtigen.

\section{DIE GRUNDLEGENDEN ZIELE DES EUREK}

Das europäische Raumentwicklungskonzept ist auf das Ziel der Europäischen Union ausgerichtet, eine ausgewogene und nachhaltige Entwicklung durch die Verknüpfung von drei politischen Zielen anzustreben:

- - wirtschaftlicher und sozialer Zusammenhalt;

- $\quad$ - Erhaltung der natürlichen Lebensgrundlagen und des kulturellen Erbes;

- $\quad$ - ausgeglichenere Wettbewerbsfähigkeit des europäischen Raumes.

Das Primat der Nachhaltigkeit zieht sich durch die gesamte Konzeption des EUREK, die Ziele sollen gemeinsam von den europäischen Institutionen sowie den nationalen, regionalen und lokalen Regierungs- und Verwaltungsebenen erreicht werden. Das EUREK ist ein Dokument, das in einem bottom-up Prozess im Zuge einer intensiven Diskussion der Mitgliedsstaaten untereinander und der Mitgliedsstaaten mit der Europäischen Kommission entstanden ist. Das Dokument ist für die Mitgliedsstaaten nicht verbindlich, es beruht auf dem Subsidiaritäts-Prinzip, wird von jedem Land insofern berücksichtigt, als es die europäischen Raumentwicklungsaspekte in seine nationale Raumordnungspolitik einbringen wird. 


\section{DER EINFLUSS DER POLITIKEN AUF DAS TERRITORIUM DER EU}

Die Weiterentwicklung der europäischen Verträge hat dazu geführt, dass die Fachpolitiken der Gemeinschaft stärkeren Einfluss auf die Raumentwicklung in den Mitgliedsländern ausüben. Vornehmlich zu erwähnen sind dabei die

Wettbewerbspolitik der Gemeinschaft;

Transeuropäische Netze (TEN);

- die Strukturfonds;

- die gemeinschaftliche Agrarpolitik;

- die Umweltpolitik;

- die politischen Dimensionen von Forschung, Technologie und Entwicklung;

- die Tätigkeit der europäischen Investitionsbank.

Ohne näher auf die einzelnen Politiken eingehen zu wollen, sei festgehalten, dass insbesondere die Strukturpolitik mit ihren Interventionen höchste Raumwirksamkeit ausübt. Ebenso gilt dies für die Verbesserung der Infrastruktur, insbesondere im Verkehrs- und Energiebereich, für den Bereich des Umweltschutzes, in dem bestimmten Räumen entsprechende Schutzkategorien zugeordnet werden, sowie für die integrierten räumlichen Entwicklungsansätze. Insbesondere sind LEADER als Politik der integrierten Entwicklung ländlicher Räume und INTERREG als Gemeinschaftsinitiative transnationaler Zusammenarbeit in der Regionalentwicklung zu nennen.

\section{POLITISCHE ZIELE UND OPTIONEN}

Die umfassende Darstellung der politischen Ziele und Optionen für das Territorium der EU sei im Folgendem kurz und insbesondere auf die grenzüberschreitenden Kooperationen reflektiert.

Die raumentwicklungspolitische Strategie, basierend auf der Einschätzung der künftigen Entwicklung der Europäischen Union, muss ein polyzentrisches Entwicklungsmodell und eine neue Beziehung zwischen Stadt und Land verfolgen. Dadurch soll eine übermäßige Konzentration von Wirtschaftskraft und Bevölkerung in wenigen herausragenden Bereichen verhindert werden. Neben dem Kernraum der EU, der von den Metropolen London, Paris, Mailand, München und Hamburg begrenzt wird, der hochwertige globale Wirtschaftsfunktionen und Dienstleistungen sowie ein hoch entwickeltes Einkommensniveau und eine bestens entwickelte Infrastruktur erreicht hat, gibt es noch weitere isolierte Wachstumsinseln. Diese 
haben allerdings noch keine im Weltmaßstab herausragende wirtschaftliche Funktion erreicht. Die Ausrichtung auf eine eher polyzentrische Gestaltung des EU-Raumes ergibt eine neue Perspektive auch für periphere Gebiete. Ziel ist die Schaffung von mehreren dynamischen Zonen weltwirtschaftlicher Integration, die gut verteilt und miteinander vernetzt, international gut erreichbar sind und aus Metropol-Regionen sowie daran angebundenen Städten und ländlichen Gebieten unterschiedlicher Größen bestehen sollen. In diesen Regionen spielen hochwertige, globale Dienstleistungen sowie ökonomische Cluster eine wesentliche Rolle.

Dezentrale Siedlungsstrukturen, abgestufte Rangfolgen von Städten, die über das gesamte Gebiet der Europäischen Union gezogen sind, stellen die Voraussetzungen für eine ausgewogene und nachhaltige Entwicklung von Regionen dar und sind der eigentliche Standortvorteil der Europäischen Union gegenüber anderen großen Weltwirtschaftsregionen. Integrierte Raumentwicklungsstrategien für Städtesysteme in den Mitgliedsstaaten sowie transnationale und grenzübergreifende Kooperationen unter Einbeziehung von Städtesystemen und deren entsprechenden ländlichen Räumen sind $\mathrm{zu}$ fördern. Dies bedingt die fachliche Zusammenarbeit bei der Raumentwicklung im Rahmen grenzübergreifender Netzwerke. Städte und Regionen außerhalb der globalen Integrationszonen und Metropolregionen können nur dann wettbewerbsfähig sein, wenn deren wirtschaftliches Potential genutzt wird.

Städte-, Verkehrsachsen und Verkehrsknoten, Messe- und Ausstellungsstädte, sowie kulturelle Zentren und kleinere Städte, die als Regionalpole die Revitalisierung der sie umgebenden ländlichen Regionen zur Aufgabe haben, insbesondere aber die Vorteilsregionen für transnationale Kooperationen gehören $\mathrm{zu}$ diesen Beispielen. Dabei spielt die nachhaltige Stadtentwicklung, basierend auf der Agenda 21 und umgesetzt durch eine multisektorale integrierte Stadtentwicklungsstrategie eine besondere Rolle. „Global denken und lokal handeln“ ist das Schlagwort.

Die neue Partnerschaft zwischen Stadt und Land basiert auf der Tatsache, dass in vielen ländlichen Gebieten der Strukturwandel bis heute nicht entsprechend vollzogen werden konnte. Ursachen für diese Strukturschwächen sind niedrige Bevölkerungsdichte, fehlende oder unzureichende Verkehrsinfrastrukturen, strukturelle Entwicklungsbrüche, ungünstiges Branchen-Mix, schlechter werdende landwirtschaftliche Produktionsbedingungen. Viele dieser Probleme sind ohne eine integrierte Sichtweise von Stadt und Land nicht mehr lösbar, Partnerschaften zwischen Stadt und Land bedürfen der Kooperation und Koordination auf unterschiedlichen räumlichen Ebenen, auf einer regionalen, überregionalen, interregionalen sowie einer transnationalen Ebene. Interregionale und transnationale Zusammenarbeit soll insbesondere durch gemeinsame Projekte und Erfahrungsaustausch erfolgen, Kooperation soll auf dem Gebiet der Komplementarität beruhen. 
Bedeutend für die Erreichung dieser Strukturen sind zwei weitere Ziele, die im EUREK formuliert worden sind:

1. Der gleichwertige Zugang zu Infrastruktur und Wissen soll durch einen integrierten Ansatz zur Verbesserung der Verkehrsanbindungen sowie durch ein polyzentrisches System der Erreichbarkeit realisiert werden. Besonderes Augenmerk gilt dabei der Kooperation zwischen den Verkehrspolitiken auf EU, nationaler und regionaler Ebene sowie in Kooperation mit den Beitrittskandidaten-Ländern.

2. Zweite wesentliche Strategie ist ein umsichtiger Umgang mit der Natur und dem Kulturerbe, wobei beide Faktoren als wichtige Entwicklungsgüter angesehen werden. Dabei ist der Weiterentwicklung europäischer ökologischer Netzwerke, wie durch NATURA 2000 vorgeschlagen, ebenso Augenmerk zu schenken, wie der grenzüberschreitenden Verbindung von Schutzgebieten, ökologisch wertvollen Gebieten und umweltsensiblen Bereichen. Der Umgang mit Kulturlandschaften und dem kulturellen Erbe soll insbesondere durch die Erhaltung und kreative Weiterentwicklung von Kulturlandschaften mit kulturhistorischer, landschaftsästhetischer und ökologischer Bedeutung gekennzeichnet sein. Integrierte Raumentwicklungsstrategien sollen Kulturlandschaften entsprechend in Wert setzen, den Schutz des kulturellen Erbes sicherstellen, und dazu beitragen, dass aktuelle Stadt- und Raumentwicklungspolitik zum kulturellen Erbe zukünftiger Generationen beiträgt.

\section{DIE ANWENDUNG DES EUREK}

Die Kooperation ist der Schlüssel für eine integrierte Raumentwicklungspolitik und ist gleichzeitig der Vorteil gegenüber isolierten Sektorpolitiken. Nationale Grenzen und administrative Hürden dürfen keine Entwicklungsengpässe darstellen. Das EUREK ist als Rahmen für die integrierte Anwendung politischer Optionen gedacht, die Ausführung ist nicht Aufgabe einer Behörde, sondern liegt in der Verantwortung von unterschiedlich strukturierten Raumentwicklungsinstitutionen und Fachplanungen. Die vertikale Kooperation besteht aus der Gemeinschaftsebene, der transnationalen und nationalen Ebene sowie der regionalen Ebene. Die horizontale Zusammenarbeit reicht vom Europarat über die Europäische Union bis zur internationalen Kooperation. Festgestellt werden muss, dass das EUREK kein Rahmenplan ist der über anderen Politikfeldern steht, seine Anwendung beruht auf dem Prinzip der Freiwilligkeit, der Kooperation, dem Konsensstreben und diversen Konsultationen der betreffenden politischen und administrativen Organe auf 
unterschiedlichen Ebenen. Erfolgreiche Raumentwicklungspolitik hängt vom Zusammenwirken von Akteuren auf unterschiedlichen räumlichen Ebenen ab, transnationale und grenzübergreifende Aktionen sind unabdingbar.

Nachhaltige Entwicklung von Grenzregionen bedarf des gegenseitigen Konsenses beiderseits der Grenzen, politischer Vereinbarungen, zwischenstaatlicher Bewertungen räumlicher Effekte und Anpassungen nationaler Gesetzgebungen. Besondere Bedeutung kommt im EUREK der Kooperation mit den Kandidaten Ländern aus Zentral- und Osteuropa zu. Es ergibt sich ein neuer Bezugsraum für den Prozess der Weiterentwicklung des europäischen Raumentwicklungskonzeptes. Es ist vor der Erweiterung notwendig, das Bewusstsein für spezifische Herausforderungen des Erweiterungsraumes $\mathrm{zu}$ verbessern. Auch wenn die Raumplanung keine ausdrückliche Gemeinschaftsaufgabe ist, so muss sie - auf Grund des finanziellen Engagement in den Beitrittsstaaten dafür sorgen, das Entwicklungen nicht kontraproduktiv werden, sondern aufeinander möglichst gut abgestimmt sind. Räumliche Abstimmung ist insbesondere bei der Planung zum Ausbau der transnationalen Verkehrsnetze, der Maßnahmen zu ökologischen Sanierung sowie bei Maßnahmen zur Strukturanpassung in ländlichen Räumen notwendig. Durch bestmögliche grenzübergreifende Kooperationen kann der Integrationsprozess wesentlich unterstützt werden. Eines der wichtigsten zu lösenden Probleme ist, das schwach ausgeprägte oder fehlende politisch administrative System zur Realisierung einer gemeinsamen Raumentwicklung und kooperativen Regionalpolitik in den mitteleuropäischen Beitrittsstaaten (partnerschaftliche Institutionen, regionale Entwicklungskonzepte). Daraus ergeben sich die vordringlich zu lösenden Probleme:

- Übergangssituation im politisch administrativen System,

- rasante wirtschaftliche Aufholprozesse, allerdings mit deutlichen räumlichen Polarisierungen,

- $\quad$ eine sich langsam und ungleichmäßig entwickelnde technische Infrastruktur,

- Umweltschäden bedeutenden Ausmaßes,

- geringe finanzielle Ressourcen der öffentlichen Hand.

Daher ist es insbesondere notwendig, die Einbindung der Beitrittsstaaten in die Europäische Raumentwicklung $\mathrm{zu}$ forcieren und diese als eine der zentralen Aufgaben anzusehen. Durch diese Kooperation wird ein Beitrag zur Vorbereitung, Begleitung, Förderung und Bewältigung des EU-Erweiterungsprozesses geleistet. 


\section{EINIGE ÜBERGEORDNETE GEDANKEN ZUR ENTWICKLUNG GRENZÜBERSCHREITENDER KOOPERATIONEN UNTER EUREK UND INTERREG III (2000-2006)}

Das EUREK ist als Leitfaden für die Politik auf transnationaler Ebene unabdingbar. Beispiele transnationaler Zusammenarbeit bei der Raumentwicklung folgen unterschiedliche Prioritäten:

- Partnerschaften zwischen Stadt und Land,

- die Entwicklung ausgewogener städtischer Systeme,

- nachhaltige Entwicklung sensibler Gebiete,

- Bereitstellung von Dienstleistungen für Haushalte und kleine und mittlere Unternehmen (KMUs),

- Forschungs- und Technologietransfer,

- Entwicklung des natürlichen und kulturellen Erbes,

- nachhaltige wirtschaftliche Aktivitäten (Tourismus).

Dabei ist insbesondere bedeutend, dass die Programme und Pilot-Aktionen sektorübergreifend angelegt sind und einen sehr hohen Grad an Zielpräzision aufweisen. Mit dem Vorliegen des EUREK-Dokumentes ist zu erwarten, dass ein dynamischer Prozess der Interaktion und gegenseitigen Bereicherung zwischen den Grundlagen des EUREK einerseits und den regionalen Visionen und Strategien andererseits erreicht wird.

Für Regionen, die grenzüberschreitend kooperieren wollen, sollte insbesondere die Erarbeitung einer gemeinsamen strategischen Vision zur Raumentwicklung Bedeutung haben, um die Umsetzung basierend auf den Entwicklungsvorgaben des EUREK bestmöglich zu gewährleisten. Dabei ist auf die Entwicklung einer eigenen Identität für den Kooperationsraum besonderer Wert zu legen. Aus den Erfahrungen europäischer Regionalentwicklung können wir drei Ansätze zur Anwendung bringen: Den horizontalen Ansatz, der versucht Prioritäten und Maßnahmen für die im EUREK enthaltenen Politikoptionen für die Region vorzuschlagen

Den konzentrierten Ansatz, der sich auf einige regionsspezifische Themen bezieht, die insbesondere auf die vorhandenen Potentiale abgestimmt sind

Den „Blick nach aussen Ansatz“, der im Kontext der globalen Wirtschaftsentwicklung Maßnahmen zur Stärkung der Wettbewerbsfähigkeit der Kooperationsregion festlegt.

In einer Phase der Intensivierung der Zusammenarbeit sollte das zentrale Ziel verfolgt werden, Partnerschaften, gemeinsame Reflexionen zu Fragen von gemeinsamen Interesse, sowie gemeinsame Arbeitsmethoden zu diskutieren. Dabei ist insbesondere die Abstimmung mit angrenzenden Gebieten, sowie mit institutionellen und 
logistischen Rahmenbedingungen zu gewährleisten.

In Überleitung zu inhaltlichen Dimensionen der Entwicklung ist insbesondere den relativen Vorteilen Rechnung zu tragen. Die Stärkung der comparative advantages sollte dazu beitragen, eine neue Identität für die Region zu entwickeln, basierend auf einem polyzentrischen Zugang. Einige Gedanken:

Kooperationen sind insbesondere anzuregen im Bereich des Tourismus, durch entsprechende Inwertsetzung natürlicher und kultureller Werte, nicht zuletzt um eine weitere Diversifizierung der Wirtschaft zu erreichen. Dies bedeutet eine Verknüpfung von Stadt und Land bei der Erstellung von Tourismuskonzeptionen.

Eine Option betrifft die Erarbeitung von Kooperationskonzepten für KMUs, sowie generell die Bereitstellung von Grundlagen, die auf eine verbesserte Nutzung von komplementären Angeboten ausgerichtet ist.

Sehr wichtig ist die Erstellung von entsprechenden institutionellen Rahmenbedingungen, die gewährleisten, dass der derzeitige „top-down“ Ansatz verstärkt eine „,bottom-up“ Dimension in den Entwicklungsprozess einbringt.

Möglichkeiten zum Austausch von Informationen sollten optimiert werden. Dabei ist zu denken an Projektbörsen, institutionalisierten Austausch von Erfahrungen im Sinne von „best practices“, ständige gemeinsame Informationsveranstaltungen, etc.

Kooperationen sollen darauf abgezielt sein, eine „kritische Masse“ für wirtschaftliche Aktivitäten und die Nutzung der Telekommunikations- und Informationstechnologien zu erreichen.

Sehr wichtig bzw. vordringlich ist die Einrichtung entsprechender Organisationsstrukturen, die nicht nur die Umsetzung der Projekte hauptverantwortlich betreuen sollen sondern auch gewährleisten sollen, dass ständig innovative Akzente bei den Projekten grenzüberschreitender Kooperationen gesetzt werden; ebenso wird eine gewisse Monitoring- und Evaluationsfunktion wahrgenommen.

Besonders entscheidend ist die Tatsache, dass bei der Abgrenzung des Kooperationsraumes eine offene Abgrenzung gewählt wird, d.h. dass programmbzw. projektbezogen unterschiedliche regionale Dimensionen Anwendung finden können und sollen. Außerdem ist besonders darauf Bedacht zu nehmen, Beziehungen mit anderen, ähnlich gelagerten Gebieten grenzüberschreitender Kooperationen zu knüpfen und auszubauen, um über einen Informations- und Erfahrungsaustausch neue Erkenntnisse $\mathrm{zu}$ gewinnen. Insbesondere ist die offene Abgrenzung des Kooperationsgebietes und die Netzwerk-Idee notwendig, um in der Folge 
Voraussetzungen für die Realisierung eines grenzüberschreitenden EUREGIOKonzeptes zu schaffen. Die Schaffung einer EUREGIO und der Erfolg derselben wird maßgeblich von der Motivation von lokaler Bevölkerung sowie potentiellen beteiligten Institutionen und Verbänden in einem bottom-up Verfahren abhängen. Für den Fall einer nachhaltigen und langfristigen Kooperation muss eine gute Organisation gebildet werden, um eine optimale Koordinierung zwischen der Europäischen Union, den Nachbarstaaten und den Institutionen bzw. Gebietskörperschaften sowie den Projektträgern zu gewährleisten.

\section{EVROPSKI KONCEPT PROSTORSKEGA RAZVOJA KOT TEMELJ PREKOMEJNEGA SODELOVANJA}

\section{Povzetek}

Prispevek poroča o ciljih in strategijah bruseljskega Evropskega koncepta prostorskega razvoja (EUREK). Eurek so k življenju obudili v maju 1999 in mu za popotnico dali podnaslov "Na poti $\mathrm{k}$ prostorsko uravnovešenemu trajnostnemu razvoju Evropske zveze". S tem so avtorji tega vizionarskega projekta opredelili obstoječi problem - t. j. divergentnost evropskih regij, obenem pa postavili smerokaze - t. j. princip trajnostno sonaravnega razvoja, ki naj usmerjajo snovalce uravnoteženega razvoja (regij).

Posebno pozornost namenja koncept prostorskemu širjenju zveze na vzhod. Prioritetne naloge vidijo snovalci EUREK v: napredovanju gospodarske integracije in kooperacije; krepitvi lokalnih in regionalnih ustanov ter njihove vloge pri načrtovanju regionalnega razvoja; in nenazadnje utrditvi prekomejnih povezav na območjih ob zunanjih mejah držav članic. Posebno pozornost, menijo, je pri tem potrebno posvetiti ureditvi zahodni Evropi kompatibilne administrativne in regionalne mreže, odpravljanju okoljevarstvenih problemov in splošni skrbi za skladnost regij neposredno ob meji.

Avtor osvetli nekatere možnosti, ki jih ta strateški koncept ponuja in priporoča skupno načrtovanje razvoja na obmejnih območjih, tako partnerskih držav Evropske zveze kot njih in kandidatk za priključitev. Prostorski načrti, ki bodo upoštevali danosti in komplementarnost območij ob meji bodo deležni pomoči, ki jo nakazuje obravnavani strateški program EU. Zato sodi v prioritetno nalogo držav in pokrajin ob zunanjih mejah zveze priprava vizij in strateških načrtov sodelovanja oziroma bodočega komplementarnega razvoja. Brez skupnih prostorskih oziroma razvojnih aktov sodelovanja ne bo mogoče voditi, nadzirati, niti podpirati. 
Kooperacije velja podpirati prednostno na področjih kjer obstaja regiji, kraju in pokrajini v EZ dopolnilna, obstoječa turistična infrastruktura na drugi strani državnih meja. Pri snovanju skupnih projektov je posebno pomembno, da potreba po sodelovanju vznikne na lokanem nivoju in ne na ravni države. Princip "Top-Down" (z vrha navzdol) naj zamenja gibalo "Bottom-Up" (iz tal v višave). Ob udejanju omenjenih navodil bo nastala naravno/družbeno pogojena prekomejna regijska povezava oziroma se bo oblikovala trdna prekomejna regija $\mathrm{v}$ smislu že zastavljenega, a prepogosto "papirnatega" koncepta Eroregij. 